\title{
Review Update on Pneumococcal Conjugate Vaccine: A New Hope for Reduction of Pneumococcal Disease in Bangladesh
}

\author{
Jannatul Fardows ${ }^{1}$, Abu Bakar Siddique ${ }^{2}$, Nasren Farhana ${ }^{3}$, Tasmine Binte Islam ${ }^{4}$ \\ ${ }^{1}$ Assistant Professor, Department of Microbiology, International Medical College, Gazipur, Bangladesh; ${ }^{2}$ Assistant Register, \\ Department of Medicine, Dhaka Medical College, Dhaka, Bangladesh; ${ }^{3}$ Lecturer, Department of Microbiology, Dhaka Medical \\ College, Dhaka, Bangladesh; ${ }^{4}$ Assistant Professor, Department of Microbiology, Tairunnessa Memorial Medical College, Gazipur, \\ Bangladesh
}

[Received: 1 April 2015; Accepted: 15 May 2015; Published: 1 June 2015]

\begin{abstract}
Pneumococcal diseases are the common and widely distributed disease in the world. It causes various mortality and morbidity every year, mainly in the developing countries. Pneumococcal conjugate vaccine (PCV) is the new vaccine against all invasive pneumococcal diseases. PCV10 and PCV13 are the available preparation in worldwide. It is safe, highly antigenic and minimal side effects. In Bangladesh, PCV is included in EPI schedule from April, 2015. The current preparation is Synforix PCV10, which is given intramuscularly with pentavalent vaccine in EPI schedule. In conclusion, the assessment of PCV impact in Bangladesh requires national and regional level surveillance and introduction of PCV in EPI will reduce the mortality and morbidity of pneumococcal disease. [Bangladesh J Infect Dis 2015;2(1):1922]

Keywords: Pneumococcal disease; PCV10; PCV13; world health organization; EPI

[How to Cite this article: Fardows J, Siddique AB, Farhana N, Islam TB. Review Update on Pneumococcal Conjugate Vaccine: A New Hope for Reduction of Pneumococcal Disease in Bangladesh. Bangladesh J Infect Dis 2015;2(1):19-22]

Corresponding author: Dr. Jannatul Fardows, Assistant Professor, Department of Microbiology, International Medical College, Gazipur, Bangladesh; Cell no.: +8801718387401; Email: jannat2026@gmail.com

Conflict of Interest: The Authors declare that there is no conflict of interest regarding the publication of this paper.

Contributions to authors: JF, ABS, NF \& TBI have contributed from literature searching, preparing and revising the manuscript.
\end{abstract}

\section{Introduction}

Pneumonia is the leading global cause of childhood death, outside of the neonatal period, and contributes to $19 \%$ of the 10 million childhood deaths occurring annually ${ }^{1}$. Pneumococcal disease is the name given to a group of diseases caused by a bacterium called Streptococcus pneumoniae, (also known as pneumococcus). Pneumococcal infection and disease can affect a variety of organ systems resulting in a number of disease syndromes. Diseases caused by pneumococcus include severe diseases such as pneumonia, meningitis and bacteraemia and milder diseases such as middle ear infection (otitis media), sinusitis and bronchitis ${ }^{2}$. Pneumococcus is classified into a number of serotypes, based on the composition of its outer capsule. There are about 93 known serotypes whose 
prevalence varies by geographic region of the world, as well as by age. These different serotypes have varying potential to cause disease with relatively few serotypes associated with severe disease in children ${ }^{3}$.Therefore, in this review paper, the current preparation, dose schedule of PCV, contraindication and side effects are briefly explained. Various reports regarding vaccine approval by UNICEF and WHO, date of approval and assessment of PCV impact in Bangladesh also discussed.

\section{Pneumococcal conjugate vaccine $(\mathrm{PCV})$}

Pneumococcal conjugate vaccine consists of sugars (polysaccharides) from the capsule of the bacterium Streptococcus pneumoniae that are conjugated to a carrier protein. Unlike the pneumococcal polysaccharide vaccine, the pneumococcal conjugate vaccine protects children younger than 2 years of age. It protects against severe forms of pneumococcal disease, such as pneumonia, meningitis, otitis media and bacteraemia ${ }^{4}$. The first pneumococcal conjugate vaccine, a 7-valent product (PCV7) was approved in 2000 in USA (Prevnar; Pfizer [Philadelphia, PA, USA]) but no longer in use because certain non-vaccine Streptococcus pneumonia serotypes still cause significant morbidity and mortality ${ }^{5}$. Two conjugate vaccines are available since 2009 , one is 10 -valent (PCV10), the other is 13-valent (PCV13) manufactured by (Synflorix; GlaxoSmithKline [Rixensart, Belgium]) .They are effective against 1, $3,5,6 \mathrm{~A}, 7 \mathrm{~F}$, and 19A serotype ${ }^{6}$.

\section{Vaccine schedule of PCV}

For PCV administration to infants World Health Organization (WHO) recommends three (3) primary doses (the $3 p+0$ schedule) or as an alternative 2 primary doses plus a booster (the $2 p+$ 1 schedule). In choosing between the $3 p+0$ and $2 p+1$ schedule, countries should consider locally relevant factors including the age distribution of pneumococcal disease, the likely vaccine coverage, and the timeliness of the vaccine doses. If the $3 p+0$ schedule is used, vaccination can be initiated as early as 6 weeks of age with an interval between doses of $4-8$ weeks, for example at 6,10 , and 14 weeks or at 2, 4 and 6 months, along with Pentavalent (DTP-HepB-Hib) by intramuscularly . Prematurely born infants (i.e. $<37$ weeks gestation) should receive PCV at the recommended chronologic age concurrent with other routine vaccinations ${ }^{7}$.

\section{Contraindication of PCV}

There is a known hypersensitivity to a prior dose. Infants with a moderate or severe illness (temperature $\geq 39^{\circ} \mathrm{C}$ ) should not be vaccinated until their condition improves ${ }^{8}$.

\section{Side effects of PCV}

There are some local reactions reported during the administration of PCV like redness, pain and swelling as well as fever ${ }^{8}$.

\section{Co-administration with other vaccines or child health intervention}

PCV can be co-administered with other EPI vaccines, i.e. during the same visit, but with a separate syringe and in a separate injection site $^{7}$.

\section{Safety and immunogenicity of PCV13 and PCV10 over PCV7}

PCV13 (Prevnar 13) and PCV10 (Prevnar 10) consists of capsular polysaccharides from the 13 and 10 most common types that cause pneumococcal disease respectively, covalently linked to a nontoxic protein that is nearly identical to diphtheria toxin. These covalent linking to a protein renders the polysaccharide antigenic in infants and toddlers 9 . Because of their excellent immunogenicity in infants and toddlers, PCV10 and PCV13 have been recommended for infants and children in its place since 2009. PCV13 began to be recommended for use in selected high-risk adults since 2012 and for all adults $\geq 65$ years of age since $2014^{10}$.

\section{History of PCV introduction in Bangladesh}

In developing countries, Pneumococcal infections cause pneumonia, meningitis and febrile bacteraemia. Nearly half-a-million annual deaths occur in children under 5 years of age by pneumococcal infections ${ }^{11}$. The overall incidence of invasive pneumococcal disease among children less than five 5 years of age was 447 episodes per 100,000 child years, which is comparable to incidence rates found among children coming to hospitals in rural settings ${ }^{12}$. WHO recommends that PCV be included in childhood immunization programme worldwide, and recommends that countries with high childhood mortality (under-5 mortality of greater than 50 for every 1,000 live births) make its introduction a high priority ${ }^{13}$. The 73 countries are eligible to access PCV via the 
Advance Market Commitment (AMC) facility in 2007. Of these, 67 countries have access to financial support through Global Alliance for Vaccine and Immunization (GAVI) to accelerate the uptake of PCV into their national immunization programmes. Of the 73 eligible countries, GAVI has approved 46 for introduction of PCV in their national immunization programme in 2009. Bangladesh is one of the countries among these eligible countries from $2013^{14}$ and PCV is included in national immunization programme from April, 2015 in Bangladesh.

\section{Available preparation of PCV in Bangladesh}

Available preparation of PCV in Bangladesh is Synflorix PCV10, two dose preparation and its given to the children by Expanded Programme on Immunization (EPI) ${ }^{7}$.

\section{Impact of PCV on Bangladesh}

PCV has been introduced in Bangladesh from April 2015. Assessment the impact of PCV on Bangladesh requires national/regional data and epidemiological surveillance. But in Bangladesh, lack of funding and newer diagnostic tools, the proper assessment is still interrupted. However, with some external and national funding, some local surveillance sites have established.

From June, 2015 a project has been started to evaluate the impact of PCV in Bangladesh. This study takes place in Sylhet, Bangladesh, at a field site known as Projahnmo that has been conducting community-based research since 2001 . The site is a partnership between Johns Hopkins University, the Bangladeshi Ministry of Health, and a number of Bangladeshi non-governmental organizations including ICDDRB, the Child Health Research Fund. This study will establish surveillance of invasive pneumococcal disease pre- and postvaccine introduction to evaluate the effectiveness of PCV10 using an incident case-control study $\operatorname{design}{ }^{15}$.

\section{Conclusion}

In conclusion, pneumococcal invasive disease is a global burden as well as developing countries. PCV is very safe with high antigenicity with minimal side effects and it is well tolerated by infant. Proper surveillance in Bangladesh can evaluate the impact of PCV and reduce the childhood mortality and morbidity. If UNICEF/WHO and GAVI will continue their close collaboration with current suppliers to improve the balance in supply and demand to the large and developing countries, mortality and mortality caused by pneumococcus should reduced to a greater extent.

\section{References}

1. Williams BG, Gouws E, Boschi-Pinto C, Bryce J, Dye C. Estimates of world-wide distribution of child deaths from acute respiratory infections. Lancet Infect Dis 2002; 2: 2532

2. Prymula R, Peeters P, Chrobok V. Pneumococcal capsular polysaccharides conjugated to protein $\mathrm{D}$ for prevention of acute otitis media caused by both Streptococcus pneumoniae and nontypable Haemophilus influenzae: a randomised double-blind efficacy study. Lancet 2006; 367: 740-748

3. McCullers JA. Insights into the interaction between influenza virus and pneumococcus. Clin Microbiol Rev 2006; 19: 571-582

4. Robinson KA, Baughman W, Rothrock G, Barrett NL, Pass M, Lexau C, Damaske B, Stefonek K, Barnes B, Patterson J, Zell ER, Schuchat A, Whitney CG. Epidemiology of invasive Streptococcus pneumoniae infections in the United States, 1995-1998: opportunities for prevention in the conjugate vaccine era. JAMA 2001; 285: 1729- 1735 .

5. Conklin, JLD, Loo, J. Kirk TB. "Systematic review of the effect of pneumococcal conjugate vaccine dosing schedules on vaccine-type invasive pneumococcal disease among young children,'Pediatr Infec Dis J 2014; 33 (2):109-118.

6. CDC (Centers for Disease Control and Prevention). "Invasive pneumococcal disease in young children before licensure of 13-valent pneumococcal conjugate vaccineUnited States, 2007," Morbidity and Mortality Weekly Report 2010; 59 (9):253-257.

7. WHO (World Health Organization).Introduction of pneumonia vaccine PCV10, two dose presentation: A handbook for district and health facility staff, 2013. WHO, 20 Avenue Appia, 1211 Geneva, 27, Switzersland. Available at http://www.who.int/vaccine documents. (Last accessed on 21Nov, 2015).

8. Black SB, Shinefield HR, Ling S. Effectiveness of heptavalent pneumococcal conjugate vaccine in children younger than five years of age for prevention of pneumonia. Pediatr Infect Dis J 2002; 21: 810-815

9. Moore MR, Link-Gelles R, Schaffner W, et al. Effect of use of 13-valent pneumococcal conjugate vaccine in children on invasive pneumococcal disease in children and adults in the USA: analysis of multisite, population-based surveillance. Lancet Infect Dis 2015; 15:301-303.

10. Feikin DR, Kagucia EW, Loo JD, et al. Serotype-specific changes in invasive pneumococcal disease after pneumococcal conjugate vaccine introduction: a pooled analysis of multiple surveillance sites. PLoS Med 2013; 10:e1001517.

11. WHO (World Health Organization). Pneumococcal conjugate vaccine for childhood immunization - WHO position paper. Wkly Epidemiol Rec 2007; 82: 93-104.

12. Abdullah Brooks W, Breiman RF, Goswami D, Hossain A, Alam K, Saha SK, Nahar K, Nasrin D, Ahmed N, El Arifeen S, Naheed A, Sack DA, Duby S. Invasive pneumococcal disease burden and implications for vaccine policy in urban Bangladesh. Am J Trop Med Hyg 2007; 77: 795-801.

13. WHO (World Health Organization). The Global Burden of Disease Project, 2012. Available June 2015 | Volume 2 | Number 1 
fromhttp://www.who.int/healthinfo/bodproject/en/index.ht ml. Last accessed on August 12, 2014.

14. UNICEF (United Nations International Childrens Emergency Fund). Pneumococcal Conjugate vaccine: Current supply and Demand outlook, 2013, NewYork. Available http://www.unicef.org/supply/index_54214.html. (Last accessed on 10 October, 2015).

15. JHSPH (Johns Hopkins Public Health) Magazine. Pneumococcal diseases in South Asia: Promise of vaccines. Johns Hopkins Public Health University, 2015; $615 \mathrm{~N}$, Wolfe street,Baltemore, MD21205 